Copyright by the American Institute of Physics (AIP). Zhou, Yuan; Apo, Daniel J.; Priya, Shashank, "Dual-phase self-biased magnetoelectric energy harvester," Appl. Phys. Lett. 103, 192909 (2013); http://dx.doi.org/10.1063/1.4829151

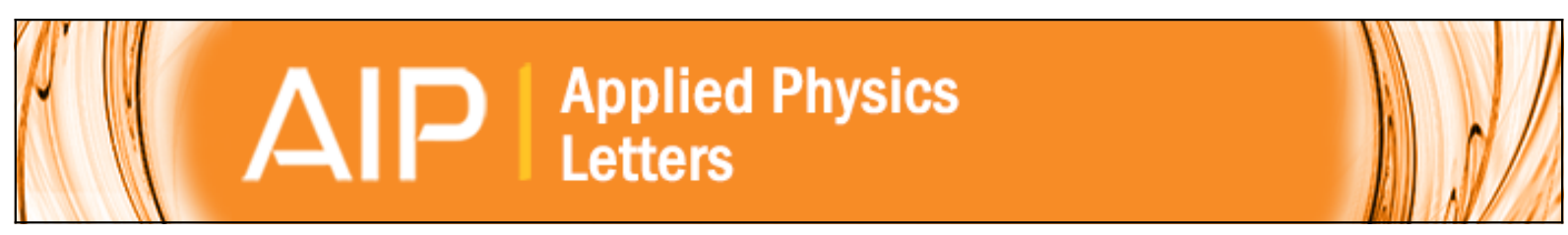

\title{
Dual-phase self-biased magnetoelectric energy harvester
}

Yuan Zhou, Daniel J. Apo, and Shashank Priya

Citation: Applied Physics Letters 103, 192909 (2013); doi: 10.1063/1.4829151

View online: http://dx.doi.org/10.1063/1.4829151

View Table of Contents: http://scitation.aip.org/content/aip/journal/apl/103/19?ver=pdfcov

Published by the AIP Publishing

\section{$\underset{\substack{\text { Anubsurus } \\ \text { Al }}}{A}$ Re-register for Table of Content Alerts}

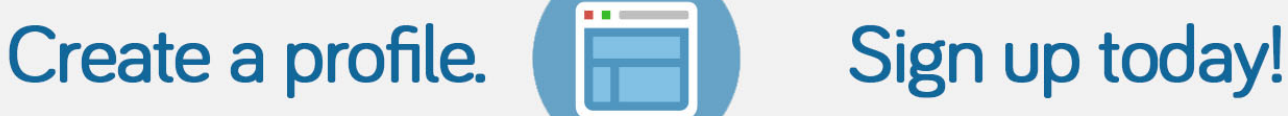

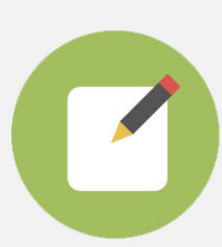




\title{
Dual-phase self-biased magnetoelectric energy harvester
}

\author{
Yuan Zhou, ${ }^{\text {a) }}$ Daniel J. Apo, and Shashank Priya ${ }^{\text {a) }}$ \\ Bio-inspired Materials and Devices Laboratory (BMDL), Center for Energy Harvesting \\ Materials and Systems (CEHMS), Virginia Tech, Blacksburg, Virginia 24061, USA
}

(Received 7 October 2013; accepted 20 October 2013; published online 6 November 2013)

\begin{abstract}
We report a magnetoelectric energy harvester structure that can simultaneously scavenge magnetic and vibration energy in the absence of DC magnetic field. The structure consisted of a piezoelectric macro-fiber composite bonded to a Ni cantilever. Large magnetoelectric coefficient $\sim 50 \mathrm{~V} / \mathrm{cm}$ Oe and power density $\sim 4.5 \mathrm{~mW} / \mathrm{cm}^{3}$ (1 $\mathrm{g}$ acceleration) were observed at the resonance frequency. An additive effect was realized when the harvester operated under dual-phase mode. The increase in voltage output at the first three resonance frequencies under dual-phase mode was found to be $2.4 \%, 35.5 \%$, and $360.7 \%$. These results present significant advancement toward high energy density multimode energy harvesting system. (c) 2013 AIP Publishing LLC. [http://dx.doi.org/10.1063/1.4829151]
\end{abstract}

Advances in integrated circuits, sensors, and actuators have led to decreased power consumption to a point where energy harvesting (EH) systems can be used to supply sufficient power to these circuits and wireless devices. ${ }^{1-3}$ Specifically, in a structural health monitoring system, there is an increasing interest in developing self-powered sensor nodes with smart architecture that utilize all the environmental resources available to meet the dynamic power requirement. ${ }^{4}$ Unused power can be tapped from environment in the form of structural vibrations (including strain and acceleration) and magnetic field available from industrial machines, transportation vehicles, human activity, and buildings. ${ }^{5,6}$ Large induction motors commonly used in industrial manufacturing plants have simultaneous presence of periodic vibrations generated by rotor and stray magnetic field from the inductive windings.

Conventional methods for vibration $\mathrm{EH}$ are based on the electromagnetic, electrostatic, piezoelectric, and magnetostrictive mechanisms. ${ }^{5,6}$ Among them, the piezoelectric mechanism shows relatively higher power density, ${ }^{7}$ however, piezoelectric harvesters are accompanied by a narrow bandwidth or a limited operating frequency range around the center operating frequency. Piezoelectric unimorph and bimorph cantilever based structures are widely used in vibration energy harvesters as they can provide low operating frequency that can be tuned by adding the tip mass. ${ }^{7,8}$ However, the power density available from the cantilever based structures is comparatively small at the $\mathrm{cm}$-scale. ${ }^{9}$ Electromagnetic harvesters operating on the principle of Faraday's law are able to provide higher power densities at larger dimensions. ${ }^{9-12}$ Therefore, to enhance the power density, researchers have made an attempt at combining the cantilever structure with moving magnetic tip mass that oscillates within an inductive core. ${ }^{9}$ Multimode energy harvesting devices with various configurations that combine electromagnetic and piezoelectric mechanism have been proposed. ${ }^{12,16,17}$ However, under small magnetic fields, the power generated from the electromagnetic energy harvester is limited. ${ }^{13}$ An alternative method to harvest magnetic

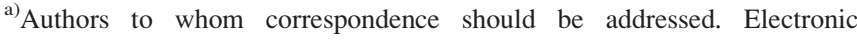
addresses: yzhou6@vt.edu and spriya@vt.edu
}

energy is by utilizing magnetoelectric composite. ${ }^{14}$ By selecting high performance piezoelectric and magnetostrictive materials and by optimizing the composite structure, it is expected that a high efficiency magnetoelectric (ME) transducer with large voltage output under low magnetic field can be obtained. ${ }^{15}$ However, to obtain the maximum ME voltage output, an optimized magnitude of DC magnetic bias is needed which presents another challenge in implementation. Alternative approaches have been suggested for magnetoelectric energy harvester that rely on the combination of highpermeabililty magnetostrictive material laminated with piezoelectric plate. ${ }^{10,11,18}$ However, in these combinations, solenoid and/or permanent magnet is required, which dramatically increases the size and increases the electromagnetic noise. Thus, even though these structures are intriguing for research, their implementation for realization of practical power sources will be limited.

In addressing these issues, we propose here a dual-phase (vibration and magnetic) energy harvesting system utilizing self-biased magnetoelectric ${ }^{19}$ response. High magnetoelectric voltage output was found to be generated from the proposed structure under the applied AC magnetic field $\left(H_{\mathrm{ac}}\right)$ in the absence of DC bias $\left(H_{\mathrm{dc}}\right)$. The voltage output was further enhanced with the co-existence of external vibration.

The dual-phase energy harvester was designed by combining magnetoelectric laminate [Figure 1(a)] and piezoelectric unimorph [Fig. 1(b)] structure in a cantilever configuration. The schematic design of the $\mathrm{EH}$ is illustrated in Fig. 1(c). The prototype consisted of a magnetostrictive/ piezoelectric $(\mathrm{M} / \mathrm{P})$ laminate structure fabricated by using a MFC (dimension $40 \times 10 \mathrm{~mm}^{2}$, Modal: M-4010-P1, Smart Material Corp., USA) and a layer of 0.25 -mm-thick Ni beam (dimension $80 \times 10 \mathrm{~mm}^{2}$ ). The composite was laminated by using epoxy resin (West System, USA). A series of Ni disks were added as tip mass $(1.1 \mathrm{~g})$ at the free end of cantilever for dynamic force excitation at the mechanical resonance. The Ni beam plays several important roles in this configuration: (i) A magnetostrictive phase in the ME laminate, (ii) a magnetic-field-active cantilever for the piezoelectric bender, (iii) a ferromagnetic phase with low field magnetic hysteresis and non-zero piezomagnetic coefficient 
that is essential for self-biased magnetoelectric response. ${ }^{19}$ When placed in an AC magnetic field, the magnetostrictive layer responds by elongating or contracting, thereby, straining the piezoelectric layer that results in output voltage across the electrical load. Under the external vibration, oscillation of the cantilever can directly create strain in the piezoelectric layer and therefore produce electrical voltage. In the absence of the applied DC magnetic field, large ME voltage can still be obtained via the self-biased ME effect. Thus, by incorporating a self-biased magnetostriction layer as a substrate for the piezoelectric layer, a ME harvester can be created for harnessing energy from both vibrations and AC magnetic field at the same time without the need for DC bias. The question is: "Are these effects additive?"

Fig. 1(d) illustrates the working principle of the fabricated prototype and also shows the experimental setup used for characterization. To characterize the harvester under dual-phase mode, the transducer was simultaneously excited by an AC magnetic field $\left(H_{\mathrm{ac}}\right)$ generated by the Helmholtz coil and a mechanical oscillation generated by the shaker. The ME response was measured in the L-L mode (longitudinally magnetized and longitudinally poled) configuration with sample located in the center of the Helmholtz coil. The induced ME voltage was monitored using a lock-in amplifier. For the vibration test, the magnetostrictive cantilever was mounted on a LDS shaker (Bruel \& Kjaer North America, Inc.) using a custom clamp. The mechanical excitation (acceleration, $a$, as well as frequency, f) was generated by the shaker using a function generator
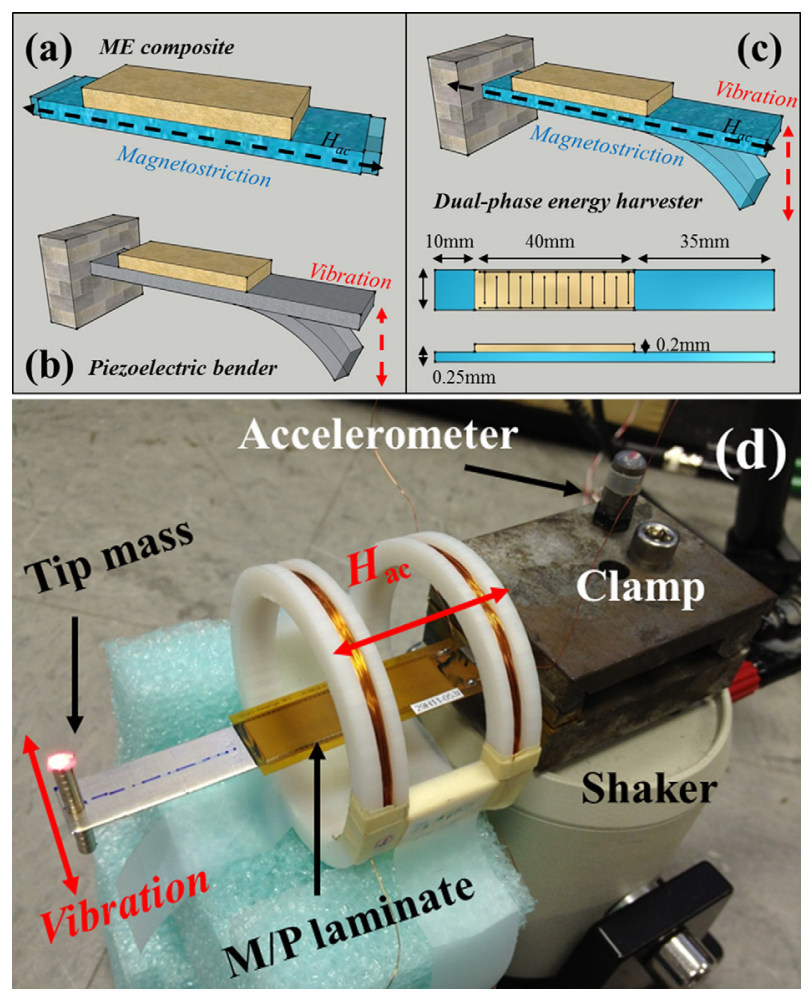

FIG. 1. Schematic diagram depicting the (a) magnetoelectric composite consisting of piezoelectric layer and magnetostrictive layer, (b) piezoelectric bender consisting of piezoelectric layer and elastic cantilever, (c) self-biased dual-phase energy harvester consisting of magnetostrictive bender and ME composite; (d) picture of the Ni-MFC dual-phase energy harvester and the experimental setup for characterization. and high power amplifier (Hewlett-Packard 6862A). The shaker base acceleration and displacement at the free end of the harvester were monitored by a low mass accelerometer (PCB U352C22) and a laser vibrometer (PDV 100, Polytech, Inc.). All the signals, including the output from laser vibrometer, power spectrum of acceleration, the transfer function from base acceleration to tip mass acceleration and the output voltage of the harvester, were collected by spectral analyzer (SigLab, Model 20-42).

We first characterized the magnetoelectric properties of the bilayer laminate as a function of DC magnetic field $H_{\mathrm{dc}}$ with $H_{\mathrm{ac}}=1 \mathrm{Oe}$ at $1 \mathrm{kHz}$ as shown in Fig. 2(a). For comparison, conventional Metglas-MFC bilayer composite with similar dimension and configuration was also fabricated and analyzed. The ME relationship for composites is given as $^{20,21}$

$$
\alpha_{\mathrm{ME}}=\left|\frac{\partial T}{\partial S} \times \frac{\partial D}{\partial T} \times \frac{\partial E}{\partial D}\right| \times \frac{\partial S}{\partial H},
$$

where $S$ is the mechanical strain, $T$ is the mechanical stress, $D$ is the electric displacement, $E$ is the electric field, and $H$ is the magnetic field. Since the first part of the above expressions is a non-magnetic factor, one can re-write Eq. (1) as

$$
\alpha_{\mathrm{ME}} \propto \frac{\partial S}{\partial H}=\frac{\mathrm{d} \lambda}{\mathrm{d} H}=q
$$

which indicates that the ME coefficient is directly related to the nature of ferromagnetic phase ( $\lambda$ : magnetostriction, $q$ : piezomagnetic coefficient). As most of the ferromagnetic materials exhibit zero piezomagnetic coefficient near zero bias, ${ }^{15}$ the $\alpha_{\mathrm{ME}}$ of Metglas-MFC structure increased from zero to a peak value $\left(\alpha_{\mathrm{ME}}=3.2 \mathrm{~V} / \mathrm{cm} \mathrm{Oe}, H_{\mathrm{dc}}=12.5 \mathrm{Oe}\right)$ at the optimum bias and then decreased. The need of optimum DC magnetic bias to achieve maximum $\alpha_{\mathrm{ME}}$ requires permanent magnet or electromagnet as external magnetic field source which increases device size and electromagnet interference. However, the $\alpha_{\mathrm{ME}}$ of Ni-MFC bilayer shows a hysteretic behavior during $H_{\mathrm{dc}}$ sweep (anticlockwise direction) with a large response of $\sim \pm 1.25 \mathrm{~V} \mathrm{~cm}^{-1} \mathrm{Oe}^{-1}$ at zero DC magnetic bias, $\sim 98 \%$ of the maximum $\alpha_{\mathrm{ME}}$ value $\left(1.38 \mathrm{~V} \mathrm{~cm}^{-1} \mathrm{Oe}^{-1}\right)$. This results from the combination of the hysteretic behavior of magnetostriction and demagnetization effect. ${ }^{19}$ It should be noted that alternative approaches have been suggested for the case of self-biased ME response that rely on the built-in magnetic bias through ferromagnetic graded structure ${ }^{22,23}$ and angular dependence of exchange bias field. ${ }^{24}$ However, in comparison with the bilayer Ni-MFC structure, these other self-biased configuration are rather complex in fabrication and implementation.

Next in order to study the resonance enhanced ME coupling, we investigated the frequency dependence of selfbiased ME performance in Ni-MFC laminate. Under zerobiased condition $\left(H_{\mathrm{dc}}=0 \mathrm{Oe}\right)$, resonance peaks with improved ME voltage output corresponding to bending modes were observed, as shown in Fig. 2(b). It should be noted that even though there is no applied $H_{\mathrm{dc}}$, self-biased ME response follows the same trend and exhibits enhanced magnitude of voltage coefficient at resonance frequency. ${ }^{19}$ 

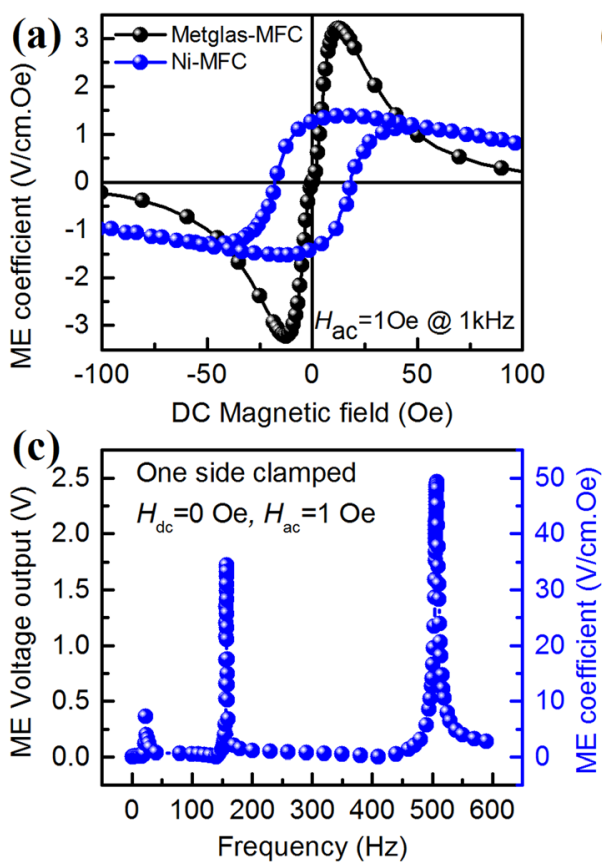

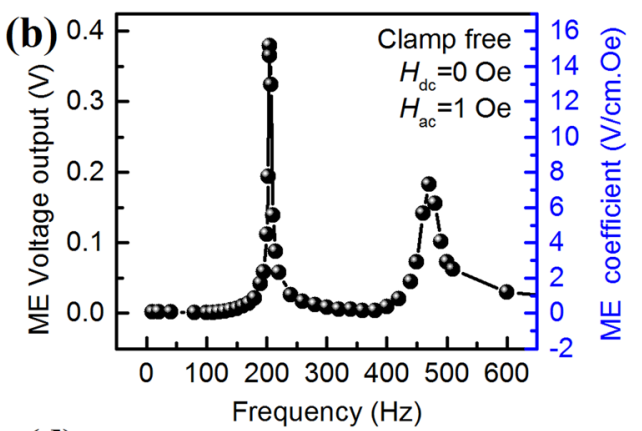

(d)

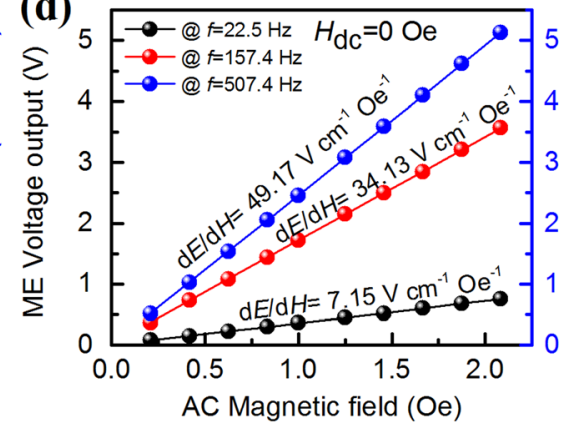

FIG. 2. Magnetoelectric response from the as-fabricated Ni-MFC self-biased magnetoelectric composite: (a) $\mathrm{ME}$ coefficient of Metglas-MFC and NiMFC bilayer laminates as function of DC magnetic field; (b) ME voltage output as a function of applied AC magnetic field frequency for the selfbiased ME composite under clamp free condition. Magnetoelectric response of the dual-phase energy harvester consist of magnetostrictive $\mathrm{Ni}$ cantilever and Ni-MFC composite: (c) ME voltage output as a function of applied AC magnetic field frequency under zerobiased condition $\left(H_{\mathrm{dc}}=0 \mathrm{Oe}\right) ; \quad(\mathrm{d})$ Source free $\left(H_{\mathrm{dc}}=0 \mathrm{Oe}\right) \mathrm{ME}$ voltage output of the dual-phase EH measured at different frequency under various applied AC magnetic field.
To quantify the dual-phase operation, the ME laminate was clamped at one end and a $\mathrm{Ni}$ tip mass was attached at the free end for enhanced bending and reducing the resonance frequency. As shown in Fig. 2(c), under longitudinally excited alternative magnetic field $\left(H_{\mathrm{ac}}=1 \mathrm{Oe}\right)$, the first bending resonance was found to occur at $22.5 \mathrm{~Hz}$. The next two resonance modes occurred at $157.4 \mathrm{~Hz}$ and $507.4 \mathrm{~Hz}$, respectively. We can also notice that the ME voltage output increased with increasing frequency where the maximum ME coefficient with the magnitude of $\sim 50 \mathrm{~V} \mathrm{~cm}^{-1} \mathrm{Oe}^{-1}$ was obtained at the resonance frequency of $507.4 \mathrm{~Hz}$. In direct ME coupling, the measured coupling coefficient is the field conversion ratio between applied AC magnetic field $\left(H_{\mathrm{ac}}\right)$ and induced $\mathrm{AC}$ electric field $\left(E_{\mathrm{ac}}\right)$,

$$
\alpha_{\mathrm{ME}}=\delta E_{a c} / \delta H_{a c}
$$

Thus, to further enhance the ME voltage output, we investigated the effect of magnetic field strength $\left(H_{\mathrm{ac}}\right)$ under zero bias condition at different frequency. Fig. 2(d) shows that the ME voltage output linearly increased with increasing AC magnetic field at three longitudinal resonance frequencies. The $\alpha_{\mathrm{ME}}$ calculated from the slope was found to be $7.15 \mathrm{~V} \mathrm{~cm} \mathrm{~cm}^{-1} \mathrm{Oe}^{-1}$ at $22.5 \mathrm{~Hz}, \quad 34.13 \mathrm{~V} \mathrm{~cm} \mathrm{~cm}^{-1} \mathrm{Oe}^{-1}$ at $157.4 \mathrm{~Hz}$, and $49.17 \mathrm{~V} \mathrm{~cm}^{-1} \mathrm{Oe}^{-1}$ at $507.4 \mathrm{~Hz}$. These calculated values match quite well with the measured value in Fig. 2(c). Large ME voltage with the magnitude of $>5 \mathrm{~V}$ was achieved at $507.4 \mathrm{~Hz}, H_{\mathrm{ac}}=2 \mathrm{Oe}$ which can be further increased with increasing $H_{\mathrm{ac}}$. These results demonstrate that by operating the $\mathrm{ME}$ energy harvester under $\mathrm{AC}$ magnetic field, large ME voltage output can be sustained under zero DC bias condition. The performance can be further improved by increasing the magnetic field strength at resonance mode.

Next, we measured the output power from the ME harvester under only vibration excitation as a function of frequency and acceleration. For measuring the frequency dependence, the device was subjected to vibration by applying constant voltage to the shaker and varying the frequency.
Fig. 3(a) shows the transfer function between base acceleration and seismic mass acceleration measured by input of random excitation. The resonance frequencies under mechanical oscillations were found to be $23.1 \mathrm{~Hz}, 156.8 \mathrm{~Hz}$, and $500.0 \mathrm{~Hz}$, respectively, which are close to that measured by applying AC magnetic field [Fig. 2(c)]. Fig. 3(b) depicts the frequency dependence of the open circuit voltage under the acceleration of $0.17 \mathrm{~g}$. The first natural bending mode was found at $20.5 \mathrm{~Hz}$ which matches well with the data from ME response. At this operating frequency and acceleration, the optimum electric load for the ME bender was determined by measuring the voltage output and the corresponding power as function of external load,

$$
P=V_{\mathrm{RMS}}^{2} / \mathrm{R} \text {. }
$$

As shown in Fig. 3(c), the vibration induced maximum electrical power output was $168 \mu \mathrm{W}$ at a matching load of $4 \mathrm{M} \Omega$; correspondingly, the power density was calculated to be $2.9 \mathrm{~mW} \mathrm{~g}^{-1} \mathrm{~cm}^{-3}$ by accounting for the total volume ( $\sim 0.34 \mathrm{~cm}^{3}$, including the volume of $\mathrm{Ni}$ cantilever, MFC, and tip mass) of the energy harvester. This power density is quite high compared to other magnetoelectric bender with similar dimension, whose specific power density was $\sim 0.4 \mathrm{~mW} \mathrm{~g}^{-1} \mathrm{~cm}^{-3}$ under mechanical vibration. ${ }^{18}$ Fig. 3(d) depicts the increased power output as a function of acceleration magnitudes. Higher acceleration can further improve the power output. These results clearly demonstrate that a high power density can be achieved by using magnetostrictive material $\mathrm{Ni}$ as the cantilever for the piezoelectric bender system. Thus, the self-biased ME energy harvester exhibited high performance in harnessing magnetic field or vibration energy.

Since both magnetoelectric and piezoelectric effect occur through elastic coupling, we further investigated the additive effect of the harvester by exciting the bender with stray magnetic field and mechanical vibration simultaneously, namely in dual-phase mode. For comparison, open circuit voltage of the $\mathrm{EH}$ at first three resonance frequency was 

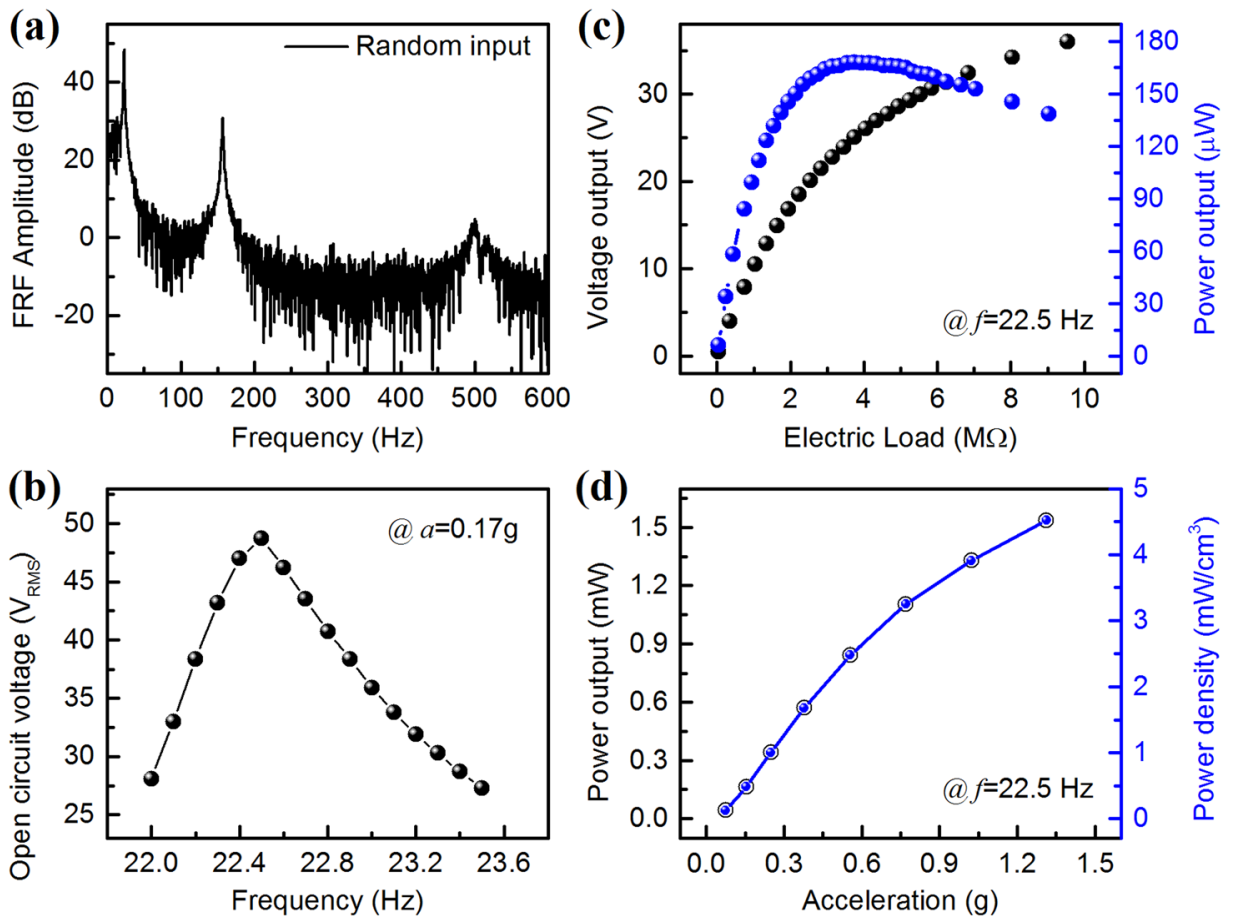

FIG. 3. Vibration mode test of the dual-phase energy harvester: (a) Base to tip-mass transfer function used to identify the nature frequency of $\mathrm{Ni}$ MFC magnetoelectric bender; (b) open circuit voltage (RMS) output of the EH as a function of frequency under vibration condition of $a=0.17 \mathrm{~g}$; (c) voltage and power output of the $\mathrm{EH}$ as function of resistive load under vibration condition of $a=0.17 \mathrm{~g}, f=22.5 \mathrm{~Hz}$; (d) Power output of the EH for various acceleration under mechanical resonance condition. measured separately under: (1) Stray magnetic field only $\left(H_{\mathrm{ac}}=1 \mathrm{Oe}\right)$, (2) mechanical vibration only $(a=0.05 \mathrm{~g})$ and (3) dual-phase $\left(H_{\mathrm{ac}}=1\right.$ Oe plus $\left.a=0.05 \mathrm{~g}\right)$ mode, as shown in Figs. 4(a)-4(c). We can clearly notice that the output voltage under dual-phase mode was improved over wide range of frequency, which implies that both power density and efficiency were enhanced through additive effect. To further quantify this combined response, the peak voltage at each single mode were added and compared with the dual-phase mode as a function of operating frequency, as shown in Fig. 4(d). In this bar chart, it is obvious that the peak voltage under dual-phase mode is close to the summation of peak voltage under each single mode, implying an additive effect was found in the dual-phase EH. The increase in voltage output at the first three resonance frequencies under dual-phase mode was found to be $2.4 \%, 35.5 \%$, and $360.7 \%$.

From these results, two important observations can be made: (1) At constant acceleration and stray magnetic field, the output voltage from the vibration is much higher at lower frequency (first bending mode) while the output voltage is higher at higher frequency under magnetic field (longitudinal resonance mode). This behavior results from the strain variation along the magnetoelectric cantilever structure due to transversal (vibration) and longitudinal (magnetostriction) excitation. (2) All the voltage response measured here are under zero-biased condition. It should be further noted here
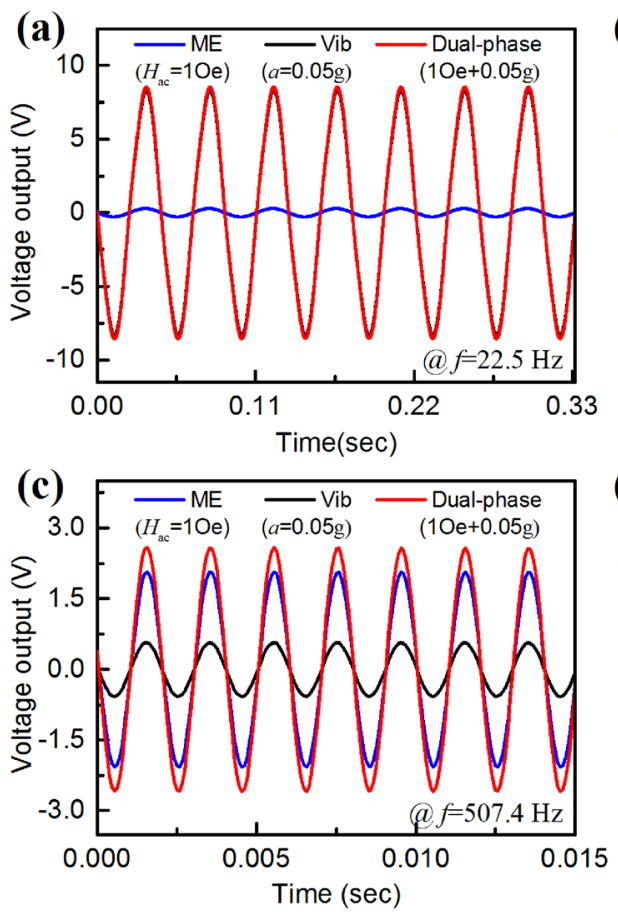
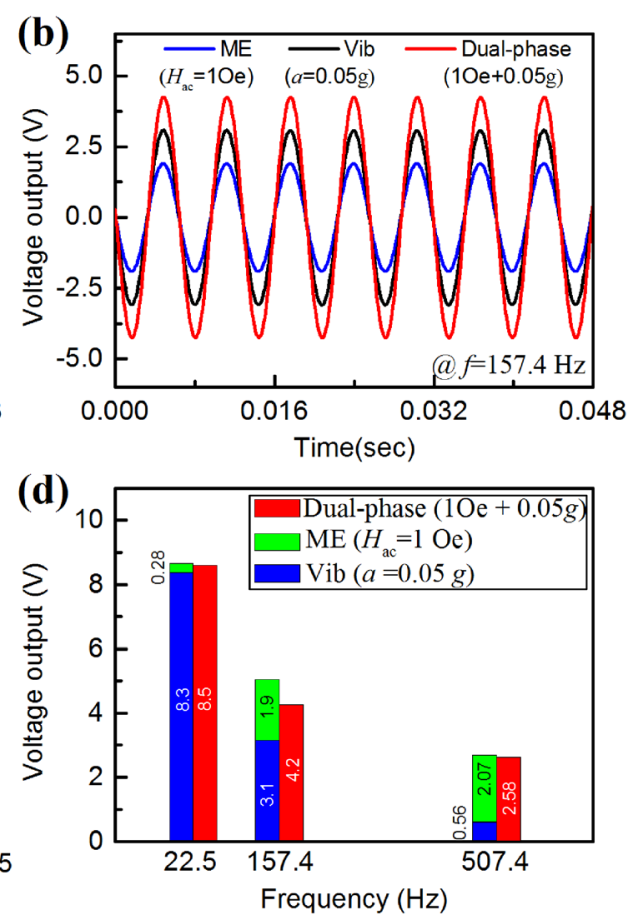

FIG. 4. Performance of the dual-phase energy harvester working under both mechanical and magnetic condition: Open circuit voltage as function of time for the $\mathrm{EH}$ under only magnetic field active condition $\left(H_{\mathrm{ac}}=1 \mathrm{Oe}\right)$, only vibration active condition $(a=0.05 \mathrm{~g})$ and the dual-phase mode $(10 \mathrm{e}+0.05 \mathrm{~g})$ at various resonance frequency (a) $22.5 \mathrm{~Hz}$, (b) $157.4 \mathrm{~Hz}$, (c) $507.4 \mathrm{~Hz}$. (d) Comparison of the EH voltage output at single mode and dual-phase mode under different driving frequency. 

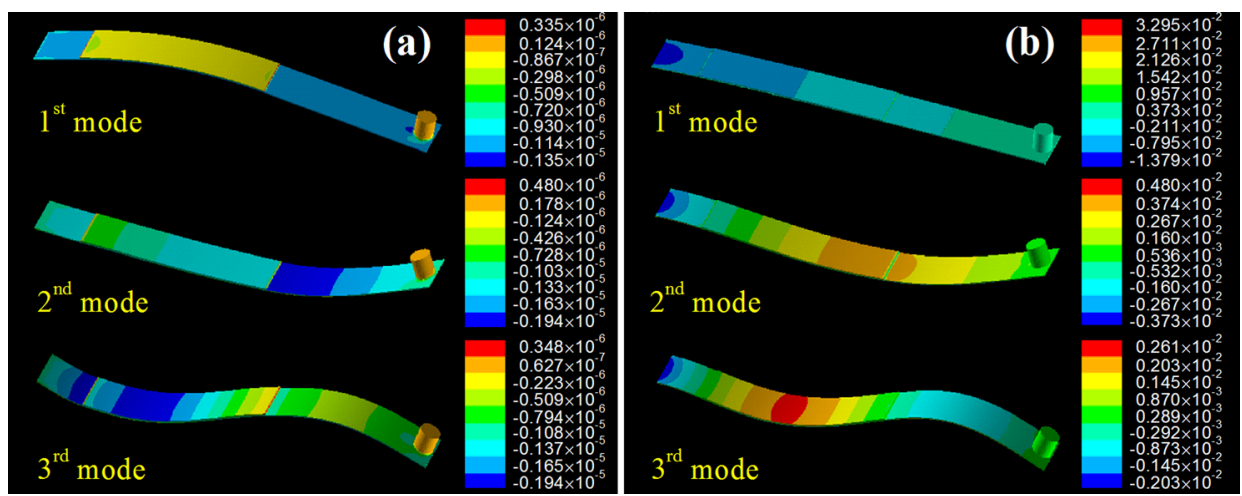

FIG. 5. FEM simulation data of strain distribution along the magnetoelectric cantilever for the first three resonance frequency (1st mode: $23.2 \mathrm{~Hz}, 2$ nd mode: $143.6 \mathrm{~Hz}$, 3rd mode: $432.7 \mathrm{~Hz}$ ): (a) Under transverse force (strain along the thickness like induced mechanical vibration), (b) under longitudinal force (strain along the length like induced magnetostriction).

that all the previous investigations on the multimode magnetoelectric harvesters have revealed that the requirement of DC magnetic field is indispensable towards enhancement of the power output. ${ }^{18,25}$

To further elucidate the performance of the EH under different (vibration/magnetic) excitations, strain distribution was simulated using the finite element model (FEM) at the first three resonance frequencies, as shown in Fig. 5. Each simulation was conducted with a maximum element mesh size of $50 \mu \mathrm{m}$ and material contact tolerance of $1 \mu \mathrm{m}$. The number of finite element nodes in the harvester was approximately $1 \times 10^{6}$. The simulated resonance frequencies for the harvester were $23.2 \mathrm{~Hz}, 143.6 \mathrm{~Hz}$, and $432.7 \mathrm{~Hz}$. Therefore, the FEM model accurately predicts the first mode but underestimates the second and third modes. The underestimation is likely due to the inhomogeneous bending behavior of the MFC, since the model does not account for imperfect bonding of the layers using epoxy. When a transverse sinusoidal force with amplitude of $1 \mathrm{~N}$ is applied to the clamped end of the beam (similar to the case of induced mechanical vibration), the MFC at higher transversal resonance mode exhibits weaker y-strain distribution (along the thickness) [Fig. 5(a)], which will result in decrease in voltage output. When a total longitudinal compressive force of $1 \mathrm{~N}$ is applied to the ends of the beam (similar to the case of induced magnetostriction), the MFC at higher longitudinal resonance mode produced a much stronger $\mathrm{x}$-strain distribution (along the length) as shown in Fig. 5(b), and a resultant increase in voltage output. These trends in strain distribution agree well with the experimental results obtained from Fig. 4(d) and imply that the higher resonance mode will generate larger strain in response to a longitudinal force. This finding is a positive step in amplifying the longitudinal or transverse strain (and thus voltage) response at a particular resonance mode.

In summary, the self-biased ME energy harvester consisting of Ni-MFC laminate and magnetostrictive Ni cantilever was fabricated and characterized. Large power density was achieved from the magnetoelectric cantilever under mechanical excitation which was further enhanced with the coexistence of AC magnetic field. The result of this study indicate that the power output of dual-phase self-biased ME energy harvester can be further improved by synchronizing the transversal (vibration) and longitudinal (magnetostriction) resonance frequency.

The authors gratefully acknowledge the financial support from the NSF INAMM program (S.P.). The authors also thank the Office of Naval Research for supporting the research (Y. Zhou) through Center for Energy Harvesting Materials and Systems.

${ }^{1}$ L. Mateu and F. Moll, Proc. SPIE 5837, 359 (2005).

${ }^{2}$ J. A. Paradiso and T. Starner, IEEE Pervasive Comput. 4, 18 (2005).

${ }^{3}$ R. Bogue, Sens. Rev. 29, 194 (2009).

${ }^{4}$ G. Park, T. Rosing, M. D. Todd, C. R. Farrar, and W. Hodgkiss, J. Infrastruct. Syst. 14, 64 (2008).

${ }^{5}$ S. P. Beeby, M. J. Tudor, and N. M. White, Meas. Sci. Technol. 17, R175 (2006).

${ }^{6}$ S. R. Anton and H. A. Sodano, Smart Mater. Struct. 16, R1 (2007).

${ }^{7}$ H. A. Sodano, D. J. Inman, and G. Park, Shock Vib. Dig. 36, 197 (2004).

${ }^{8}$ S. Roundy and P. K. Wright, Smart Mater. Struct. 13, 1131 (2004).

${ }^{9}$ Y. Tadesse, S. J. Zhang, and S. Priya, J. Intell. Mater. Syst. Struct. 20, 625 (2009).

${ }^{10}$ X. Bai, Y. Wen, J. Yang, P. Li, J. Qiu, and Y. Zhu, J. Appl. Phys. 111, 07A938 (2012).

${ }^{11}$ Y. Zhu and J. W. Zu, IEEE Trans. Magn. 48, 3344 (2012).

${ }^{12}$ X. Xing, J. Lou, G. M. Yang, O. Obi, C. Driscoll, and N. X. Sun, Appl. Phys. Lett. 95, 134103 (2009).

${ }^{13} \mathrm{P}$. Li, Y. Wen, J. Chaobo, and L. Xinshen, IEEE Trans. Ind. Electron. 58, 2944 (2011).

${ }^{14}$ S. Priya, R. Islam, S. X. Dong, and D. Viehland, J. Electroceram. 19, 149 (2007).

${ }^{15}$ Ce-Wen Nan, M. I. Bichurin, S. Dong, D. Viehland, and G. Srinivasan, J. Appl. Phys. 103, 031101 (2008).

${ }^{16}$ L. Wang and F. G. Yuan, Smart Mater. Struct. 17, 045009 (2008).

${ }^{17}$ A. Khaligh, P. Zeng, and C. Zheng, IEEE Trans. Ind. Electron. 57, 850 (2010).

${ }^{18}$ S. X. Dong, J. Y. Zhai, J. F. Li, D. Viehland, and S. Priya, Appl. Phys. Lett. 93, 103511 (2008).

${ }^{19}$ Y. Zhou, S. C. Yang, D. J. Apo, D. Maurya, and S. Priya, Appl. Phys. Lett. 101, 232905 (2012).

${ }^{20}$ K. H. Cho and S. Priya, Appl. Phys. Lett. 98, 232904 (2011).

${ }^{21}$ Y. K. Yan, Y. Zhou, and S. Priya, Appl. Phys. Lett. 102, 052907 (2013).

${ }^{22}$ S. C. Yang, C. S. Park, K. H. Cho, and S. Priya, J. Appl. Phys. 108, 093706 (2010).

${ }^{23}$ S. K. Mandal, G. Sreenivasulu, V. M. Petrov, and G. Srinivasan, Appl. Phys. Lett. 96, 192502 (2010).

${ }^{24}$ E. Lage, C. Kirchhof, V. Hrkac, L. Kienle, R. Jahns, R. Knochel, E. Quandt, and D. Meyners, Nature Mater. 11, 523 (2012).

${ }^{25}$ R. C. Kambale, W. H. Yoon, D. S. Park, J. J. Choi, C. W. Ahn, J. W. Kim, B. D. Hahn, D. Y. Jeong, B. C. Lee, G. S. Chung, and J. Ryu, J. Appl. Phys. 113, 204108 (2013). 